\title{
Finite-Aperture Wire Grid Polarizers
}

Michael A. Jensen

jensen@byu.edu

Gregory P. Nordin

nordin@byu.edu

Follow this and additional works at: https://scholarsarchive.byu.edu/facpub

Part of the Electrical and Computer Engineering Commons

\section{Original Publication Citation}

M. A. Jensen and G. P. Nordin, "Finite-Aperture Wire Grid Polarizers", J. Opt. Soc. Am. A 17(12), pp. 2191-2198 (2)

\section{BYU ScholarsArchive Citation}

Jensen, Michael A. and Nordin, Gregory P., "Finite-Aperture Wire Grid Polarizers" (2000). Faculty Publications. 1104.

https://scholarsarchive.byu.edu/facpub/1104

This Peer-Reviewed Article is brought to you for free and open access by BYU ScholarsArchive. It has been accepted for inclusion in Faculty Publications by an authorized administrator of BYU ScholarsArchive. For more information, please contact ellen_amatangelo@byu.edu. 


\title{
Finite-aperture wire grid polarizers
}

\author{
Michael A. Jensen \\ Department of Electrical and Computer Engineering, 459 Clyde Building, Brigham Young University, Provo,
} Utah 84602

Gregory P. Nordin

Department of Electrical and Computer Engineering, University of Alabama, Huntsville, Huntsville, Alabama 35899

Received May 15, 2000; accepted June 27, 2000

\begin{abstract}
The transmission characteristics of wire grid polarizers fabricated in finite apertures are investigated by using a three-dimensional finite-difference time-domain formulation. Specifically, the optical transmissivity and extinction ratio are characterized for a wide variety of geometrical parameters including aperture size in both dimensions, conducting wire fill factor, and polarizer thickness. A dispersive material model is used to investigate the performance of polarizers fabricated by using realistic metals at infrared wavelengths. The results indicate that the aperture dimension significantly impacts the polarizer transmission behavior and that the extinction of the unwanted polarization is often limited by depolarizing scattering that is due to the finite aperture size. (C) 2000 Optical Society of America [S0740-3232(00)01912-8]

OCIS codes: $050.0050,050.1970,230.0230,230.3990$.
\end{abstract}

\section{INTRODUCTION}

Wire grid polarizers have long been recognized as an effective option for discriminating between orthogonal linear polarization states in the infrared portion of the electromagnetic spectrum. ${ }^{1,2}$ Such polarizers can be fabricated by placing conducting wires within an aperture formed in a larger opaque screen. When this aperture is electrically large, the device behavior is similar to that of a polarizer of infinite extent, and its performance can be accurately characterized by using simulation approaches such as rigorous coupled-wave analysis. ${ }^{3-5}$ However, new fabrication technologies and applications that require small-aperture polarizers ${ }^{6}$ and arrays of micropolarizers ${ }^{7-9}$ have motivated the development of devices with electrically small apertures. In this case, alternative simulation techniques that model the finiteaperture extent must be explored for device characterization.

Some recent work has appeared on the subject of simulating finite-aperture diffractive devices. For example, the boundary element method has been shown to be effective in the analysis of finite-number-of-periods dielectric and finite-conductivity metallic grating structures, ${ }^{10}$ diffractive optical lenses, ${ }^{11-13}$ and other aperiodic structures. ${ }^{14}$ Method-of-moments solution techniques have also been applied to lenslet analysis based on both rigorous surface ${ }^{15}$ and volume ${ }^{16}$ integral formulations for the fields. For two-dimensional (2-D) perfectly conducting gratings, spectral-domain mode-matching solutions appear to be effective. ${ }^{17-19}$ While these studies have provided significant insight into the behavior of finite-extent diffractive devices, they have not focused on extending and applying such techniques to perform detailed analyses of finite-aperture wire grid polarizers.

In this paper we present a detailed characterization of wire grid polarizers placed in finite apertures. The analysis uses a three-dimensional (3-D) formulation of the finite-difference time-domain (FDTD) method because of its computational efficiency and geometrical and material modeling flexibility. ${ }^{20-22}$ For specific cases the FDTD results are compared with data obtained from a 2-D spectral-domain mode-matching solution technique. ${ }^{18,19,23,24}$ Additionally, because many practical metals used for micropolarizer fabrication possess permittivities with negative real parts at infrared wavelengths, a dispersive FDTD method is implemented to assess the performance of devices fabricated with these materials. ${ }^{25}$ These simulation tools are used to perform a systematic study of device performance for various geometrical and material configurations. The results indicate that the aperture size exercises a significant influence on the polarizer transmission behavior. The data further show that the polarizer extinction performance is often limited by depolarizing scattering that is due to finite-aperture effects.

\section{GEOMETRY AND ANALYSIS}

The problem under consideration is that of a plane wave impinging on a finite-sized aperture located in an otherwise infinite conducting plane of thickness $D$. As indicated in Fig. 1, the aperture is periodically loaded with equal-sized conducting wires of width $w$ and center-tocenter spacing $\Delta x$. The polarizer is assumed to be embedded in a homogeneous medium (free space for the computations in this paper), although the analysis used could be extended to allow for variations in the surrounding material parameters. For simplicity, the incident plane wave will be constrained to travel in the positive $z$ direction. With this assumption the terminology $x$ polarization and $y$ polarization will be used to designate illuminating fields that contain electric field vectors polarized in 
the $x$ direction and the $y$ direction, respectively. Throughout this paper, all dimensions will be given in terms of the illumination free-space wavelength $(\lambda)$.

\section{A. Finite-Difference Time-Domain Method}

The FDTD method is an excellent tool for analyzing the electromagnetic behavior of optical devices because of its modeling flexibility, implementation simplicity, and computational efficiency. ${ }^{26-28}$ To introduce the plane-wave excitation with minimal distortion, the FDTD implementation uses a total field/scattered field boundary ${ }^{21}$ for $z<0$. The FDTD grid is truncated by using a perfectly matched layer (PML) absorbing boundary condition ${ }^{29,30}$ that is eight FDTD cells thick $(0.40 \lambda$ thick given the FDTD cell size of $0.05 \lambda$ ). A buffer layer between the polarizer and the PML is also implemented to reduce corruption of the results due to artificial reflections from the absorbing boundary condition. The PML conductivities increase quadratically away from the computational domain, ${ }^{29}$ with the profile chosen to achieve a reflection coefficient of $10^{-5}$. The polarizer ground plane extends into the PML layer to avoid unwanted leakage of the incident fields into the region $z>D$. Figure 2 illustrates the computational configuration along with all of the relevant dimensions used for the analysis.

The simulation is performed by introducing a normally incident single-frequency plane wave into the domain and allowing the simulation to proceed until the fields in the region reach a steady-state condition. The fields on a planar surface one FDTD cell beyond the polarizer output plane are then sampled over a complete sinusoidal temporal cycle. Because of the staggered placement of the vector field components in the standard FDTD grid, spatial averaging is used to place all tangential field components at the center of each cell. The sampled fields are then transformed to the frequency domain in preparation for postprocessing evaluations of the polarizer behavior.

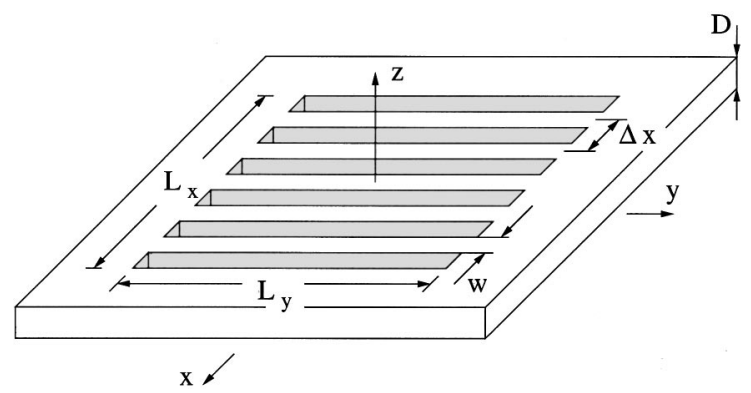

Fig. 1. Geometry for a plane wave incident on the finiteaperture wire grid polarizer.

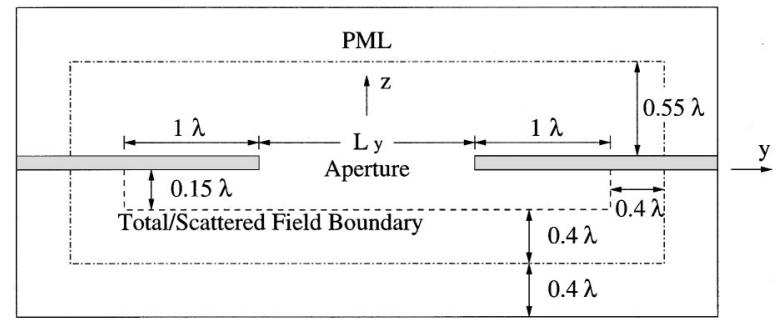

Fig. 2. Computational geometry for FDTD simulation of the finite-aperture wire grid polarizer.
Evaluating the polarizer transmissivity requires computing the real power transmitted through the device. This can be accomplished by integrating the frequencydomain sampled fields on the output plane to obtain farzone fields ${ }^{21}$ and integrating the resulting Poynting vector on a hemispherical surface. Unfortunately, this process is computationally very costly and can be inaccurate if the sampling in the far zone is not adequately dense. An improved procedure is to directly integrate the Poynting vector from the electric (E) and magnetic (H) fields on the output sampling plane. When this approach is followed, the total power transmitted by the aperture is given as

$$
P_{T}=\frac{1}{2} \operatorname{Re}\left(\int_{A} \mathbf{E} \times \mathbf{H}^{*} \cdot \hat{z} \mathrm{~d} x \mathrm{~d} y\right),
$$

where $A=L_{x} L_{y}$ is the aperture area and $\hat{z}$ denotes a unit vector in the $z$ direction. It should be recognized that any power contained in the evanescent field will be imaginary and therefore will not contribute to the value of $P_{T}$. The transmissivity is then defined as the ratio of the transmitted power to the total power intercepted by the aperture, or

$$
T=\frac{2 \eta_{0}}{A} \frac{P_{T}}{\left|\mathbf{E}^{i}\right|^{2}},
$$

where $\eta_{0}$ is the intrinsic wave impedance of the surrounding medium and $\mathbf{E}^{i}$ is the incident plane-wave electric field intensity.

Because the integration plane is very close (one cell $=0.05 \lambda$ ) to the polarizer, the fields are spatially localized near the aperture, implying that the integration needs to be performed over only a limited region. To ensure the accuracy of the integration used in this work, the value of $P_{T}$ computed for a large aperture (3.90入 $\times 3.90 \lambda$ ) was examined as a function of the size of this integration region for an $x$-polarized illuminating plane wave. This study showed that the computed power quickly converges as the integration domain is extended, with $99 \%$ of the power being captured by a region approximately $0.2 \lambda$ larger than the aperture.

\section{B. Material Model}

Most of the results presented in this paper assume that the conducting wires forming the polarizer are constructed of perfectly conducting materials. However, it is also useful to assess the effects on polarizer performance of real metals with finite conductivity. Interestingly, many practical metals used for thin-film wire fabrication, including aluminum, gold, and molybdenum, are characterized by a relative permittivity with a negative real part for infrared wavelengths. ${ }^{31}$ Physically, this implies a material response that is $180^{\circ}$ out of phase with the illuminating field. Computationally, however, this leads to instability in the FDTD simulations.

To allow FDTD analysis of these materials, a dispersive material model ${ }^{25}$ has been extended for 3-D simulations and implemented for this study. In this approach, differential equations describing the temporal evolution of the polarization current $\mathbf{J}_{p}$ and the polarization $\mathbf{P}^{L}$ generated by the material are obtained by transforming 
the Lorentz material model from the frequency to the time domain. These equations are then coupled to Maxwell's equations and are discretized in the FDTD algorithm. Prior implementations of this technique placed the polarization quantities $\mathbf{J}_{p}$ and $\mathbf{P}^{L}$ at the center of the FDTD unit cell. ${ }^{25}$ However, our observations reveal that this practice can lead to anomalous material responses that do not match reasonable physical behavior, particularly for weak transmitted field intensities. To avoid this difficulty, we have implemented the scheme by placing the vector components of $\mathbf{J}_{p}$ and $\mathbf{P}^{L}$ coincident with the electric field components in the standard FDTD grid.

\section{RESULTS}

In this section, we utilize the methodologies discussed in Section 2 to investigate the behavior of finite-aperture wire grid polarizers for different geometrical parameters. Convergence studies of the transmitted power versus the number of sinusoidal periods used in the FDTD simulations indicate that 10 and 20 cycles are required to achieve steady state for perfectly conducting and nonperfectly conducting polarizers, respectively. Throughout the discussion, we will use the following definitions:

$$
\text { fill factor }=w / \Delta x,
$$

$$
\text { extinction ratio }=T_{x} / T_{y} \text {, }
$$

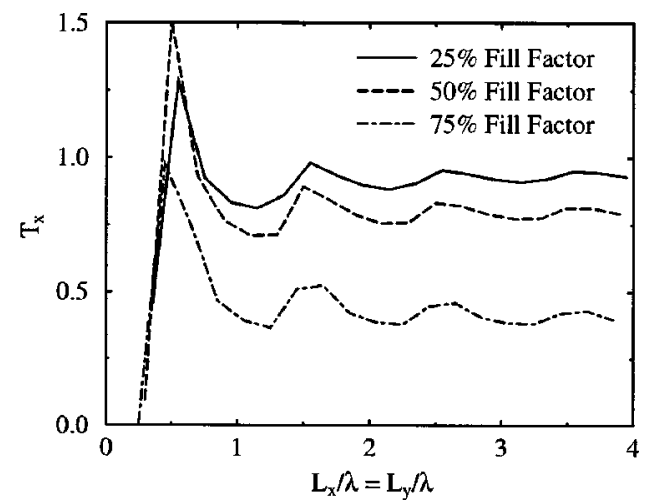

(a)

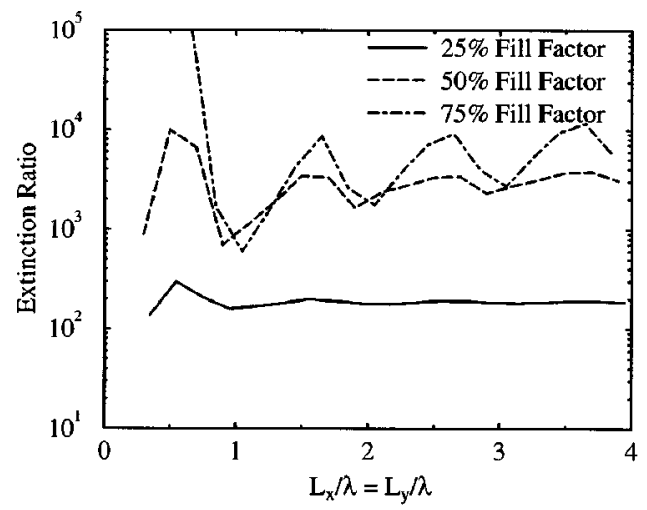

(b)

Fig. 3. (a) Transmissivity and (b) extinction ratio as a function of aperture side length for a square aperture for several values of fill factor $\left(D=0.05 \lambda, L_{x}=L_{y}\right)$.

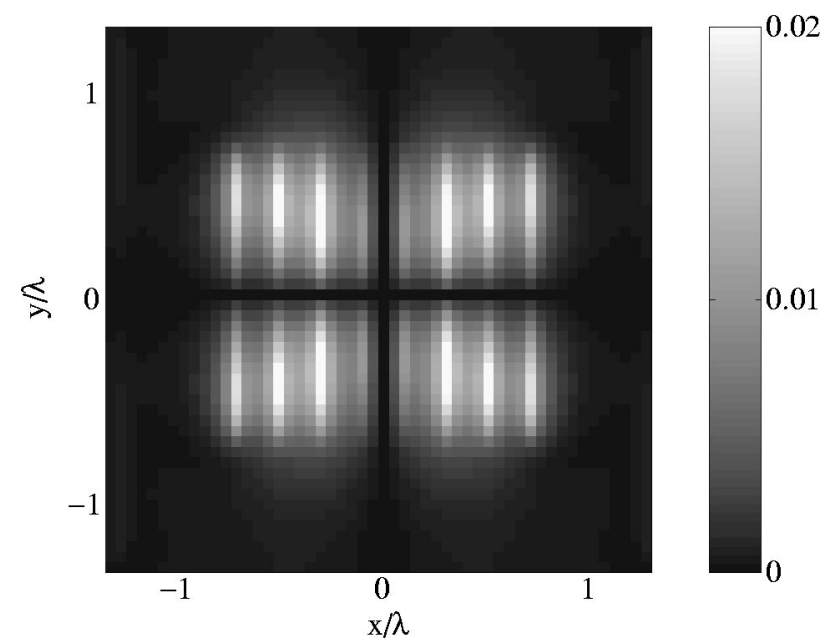

Fig. 4. Spatial map of the $x$-polarized transmitted field for a $y$-polarized incident plane wave on the square aperture of dimensions $L_{x}=L_{y}=1.45 \lambda$ and with $75 \%$ fill factor.

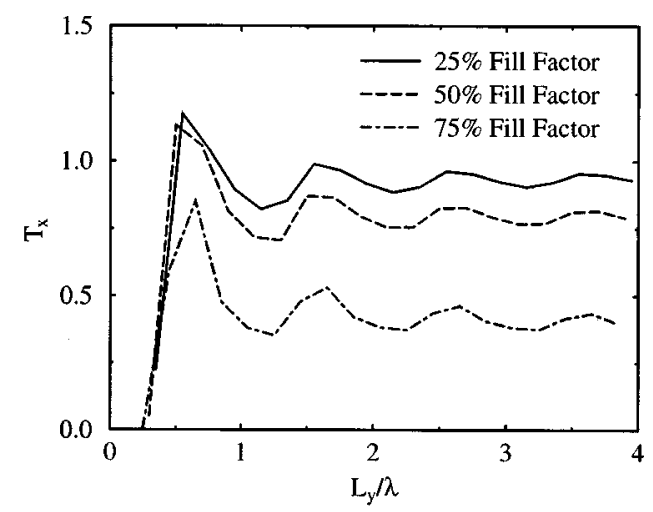

(a)

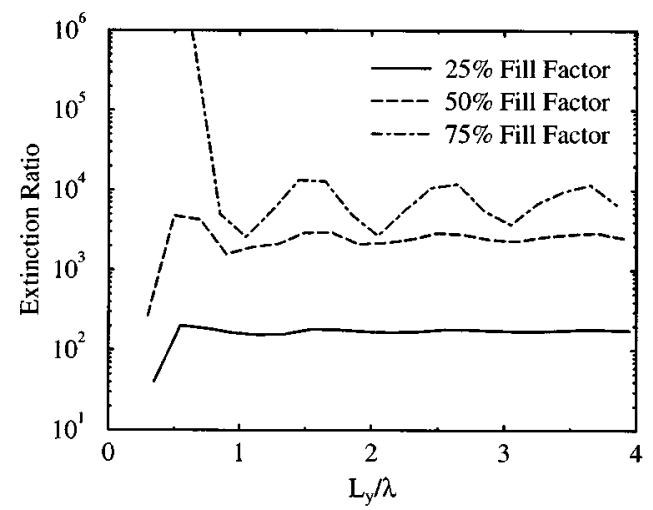

(b)

Fig. 5. (a) Transmissivity and (b) extinction ratio as a function of aperture side length $L_{y}$ for a rectangular aperture for several values of fill factor $(D=0.05 \lambda)$

where the subscripts on the transmissivity $(T)$ denote the polarization of the illuminating field. In all of the results, the wire spacing is set to $\Delta x=0.2 \lambda$. Unless otherwise noted, all reported transmissivity values are for an $x$-polarized illuminating field, since this is typically the parameter of interest in the design of practical devices (i.e., this represents the optical throughput of the polarizer if the transmissivity of the $y$-polarized light is very 
small in comparison). Also, except as noted in particular cases, the polarizer is constructed of a perfectly conducting material with a thickness of $D=0.05 \lambda$ (see Fig. 1).

\section{A. Effect of Aperture Dimension}

To begin our study, we investigate the behavior of the polarizer transmission as a function of the aperture dimensions. Consider the results in Figs. 3(a) and 3(b), which show the transmissivity and the extinction ratio, respectively, for a square aperture as a function of the aperture side length $\left(L_{x}=L_{y}\right)$. Results for three different values of fill factor are provided. It is important to recognize that in these and other plots in the paper, the disjoint plot appearance stems from the fact that the aperture dimension is increased in increments of $\Delta x=0.2 \lambda$.

Several different observations can be made from the data shown in these plots. First, it is apparent that for aperture dimensions near $\lambda / 2$, the transmissivity can actually be greater than unity as a result of the strong resonant behavior of the fields within the aperture "cavity," confirming the result of prior studies. ${ }^{18,19}$ Since the transmissivity is defined as the ratio of the transmitted power to that intercepted by the aperture, this result physically means that energy in currents surrounding the aperture edge contributes to the transmitted radiation. Second, rather than quickly converging to a final value, both the transmissivity and the extinction ratio tend to oscillate as a function of the aperture size. These peaks occur when the aperture dimension is near an odd mul-

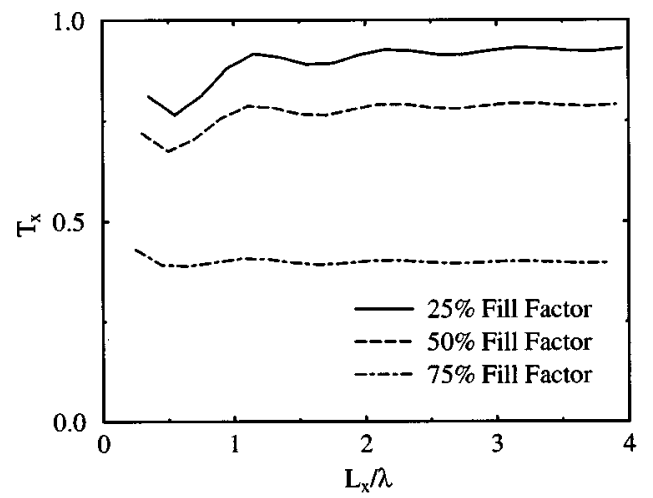

(a)

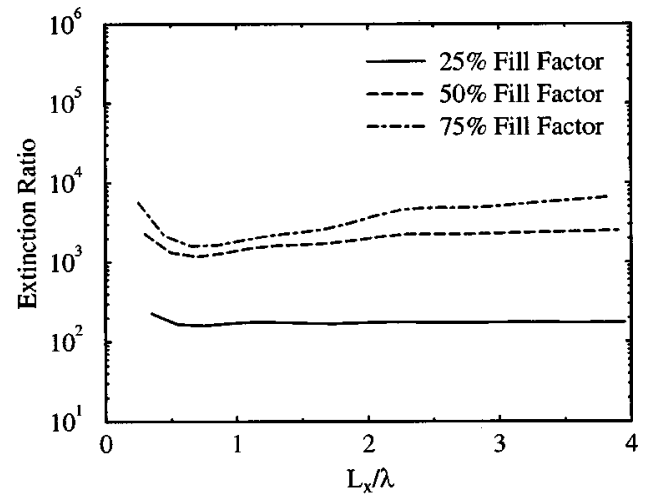

(b)

Fig. 6. (a) Transmissivity and (b) extinction ratio as a function of aperture side length $L_{x}$ for a rectangular aperture for several values of fill factor $(D=0.05 \lambda)$.

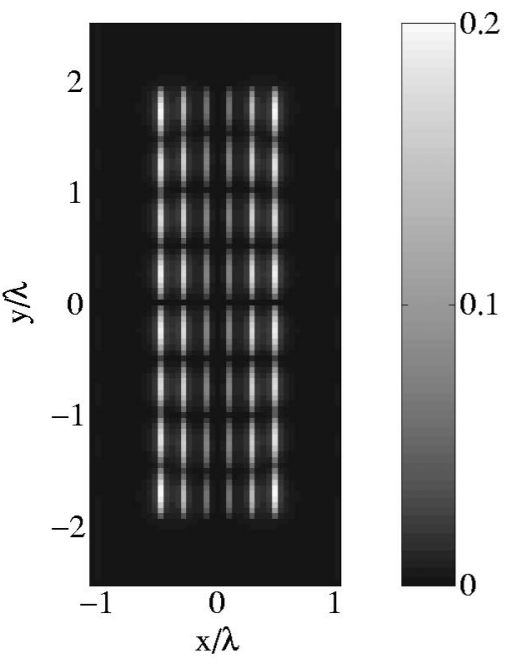

(a)

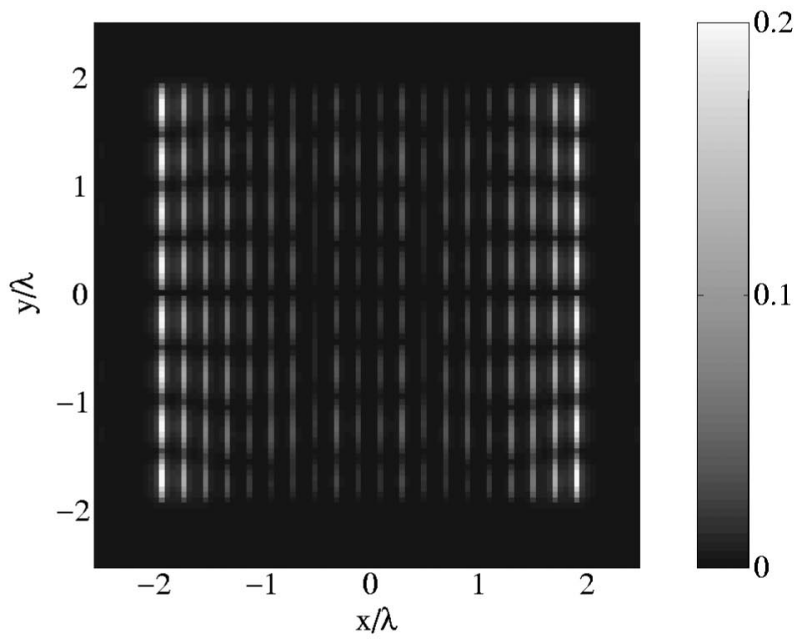

(b)

Fig. 7. $x$-polarized transmitted field strength for $y$-polarized illumination with $75 \%$ fill factor and $L_{y}=3.85 \lambda(D=0.05 \lambda)$ : (a) $L_{x}=1.05 \lambda$, (b) $L_{x}=3.85 \lambda$.

tiple of half-wavelengths, again suggesting a resonant behavior associated with the finite-aperture dimensions. Finally, as we may expect, the transmissivity decreases while the extinction ratio increases as the fill factor is increased. ${ }^{32}$ However, it is noteworthy that the increase in extinction is not commensurate with the increase in fill factor from $50 \%$ to $75 \%$. To explore this phenomenon more carefully, consider the plot in Fig. 4, which shows the magnitude of the $x$-polarized electric field component of the transmitted field for a $y$-polarized illumination field. The aperture dimensions are $L_{x}=L_{y}=1.45 \lambda$ with a $75 \%$ fill factor. For this same configuration, the maximum value of the $y$-polarized field on the integration surface is $4 \times 10^{-5}$. These results imply that for low values of fill factor, $T_{y}$ is dominated by the $y$-polarized transmission, which decays rapidly with increasing fill factor. However, as the fill factor assumes larger values, $T_{y}$ is dominated by scattering into the $x$-polarized field that is due to finite-aperture effects. Because this scattering does not decrease significantly with increasing fill 
factor, this phenomenon becomes a limiting factor in the achievable extinction of the undesired polarization.

More insight can be gained concerning the effects of the aperture dimensions by studying the polarizer behavior while varying only $L_{x}$ or $L_{y}$. Figures 5 and 6 illustrate the results when increasing the aperture in the $y$ and $x$ dimensions, respectively. In each case, the fixed dimension is chosen such that the aperture is square when the increasing dimension attains its largest value. For example, this leads to $L_{x}\left(L_{y}\right)=3.95 \lambda, 3.90 \lambda$, and $3.85 \lambda$ for fill factors of $25 \%, 50 \%$, and $75 \%$, respectively, in Fig. 5 (6).

Several additional conclusions can be drawn from the data in Figs. 5 and 6 . First, the oscillatory behavior is more pronounced in Fig. 5 than in Fig. 6, implying that resonances in the dimension parallel to the wires are more influential than those in the dimension perpendicular to the wires. Second, both Figs. 5(b) and 6(b) confirm the trend that the increase in extinction ratio is not commensurate with the increase in fill factor. Finally, we observe in Fig. 6(b) that for larger values of fill factor, the extinction tends to increase with aperture dimension. To explore this more fully, consider the plots in Fig. 7, which depict the $x$-polarized transmitted field for a $y$-polarized illumination. The polarizer has a fill factor of $75 \%$ and $L_{y}=3.85 \lambda$. The data in Figs. 7(a) and 7(b) are for $L_{x}=1.05 \lambda$ and $3.85 \lambda$, respectively. In both cases, the

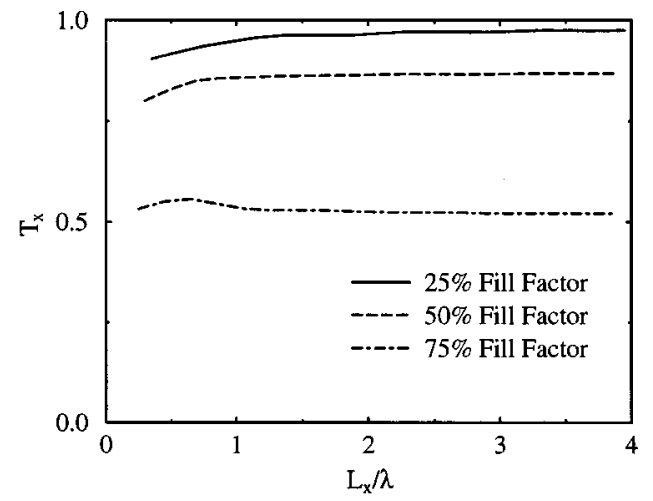

(a)

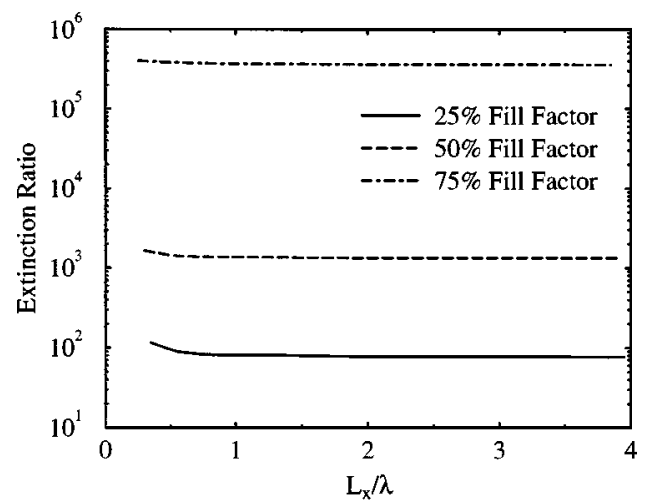

(b)

Fig. 8. (a) Transmissivity and (b) extinction ratio as a function of aperture side length $L_{x}$ for an infinitely long aperture $\left(L_{y}\right.$ $\rightarrow \infty)$ for several values of fill factor $(D=0.05 \lambda)$. These results are obtained by using the mode-matching solution technique.

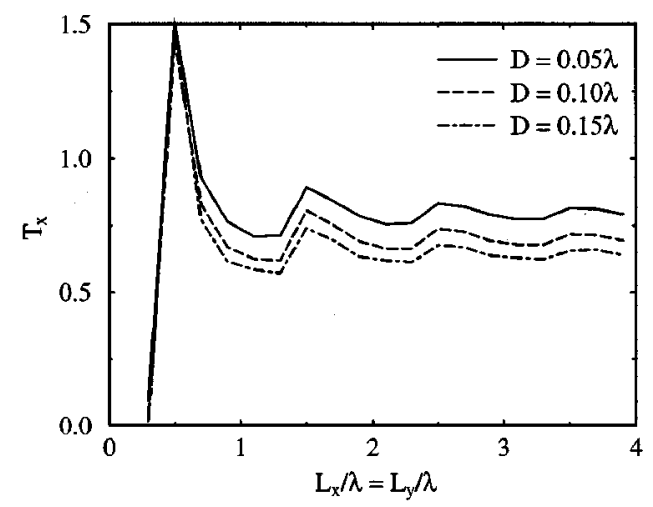

(a)

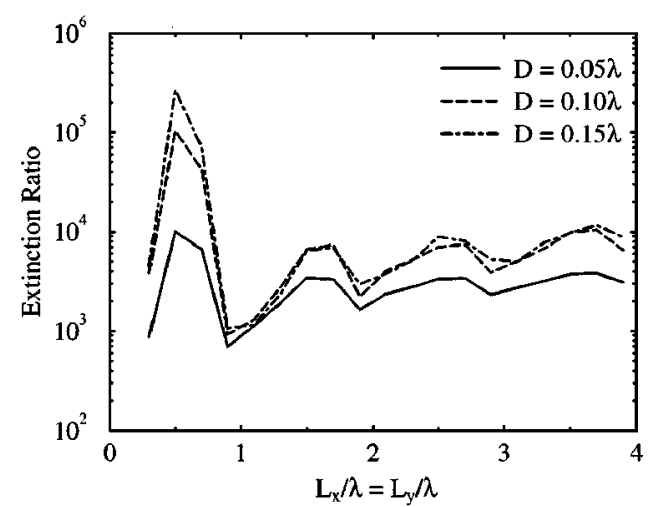

(b)

Fig. 9. (a) Transmissivity and (b) extinction ratio as a function of aperture side length for a square aperture for several values of thickness (50\% fill factor).

$y$-polarized output field strengths are below $10^{-3}$, implying that the transmissivity is dominated by the $x$-polarized fields. The key observation from these plots is that strong values of cross-polarized fields occur principally in the grooves at the edges of the aperture. As the value of $L_{x}$ increases, the relative influence of these strong field values decreases, resulting in an overall decrease in the value of $T_{y}$. This produces the increase in the extinction ratio observed in Fig. 6(b).

As a final study of the effect of aperture dimension on polarizer behavior, it is interesting to compare the FDTD results with those from a 2 -D $\left(L_{y} \rightarrow \infty\right)$ simulation procedure based on a spectral-domain mode-matching solution. ${ }^{18,23}$ Figure 8 shows the variation of $T_{x}$ and the extinction ratio with increasing values of $L_{x}$ for the three different values of fill factor. Comparison of these results with those in Fig. 6 reveals that the two methods predict very similar behaviors, although there are notable discrepancies. First, the computations indicate that the FDTD method predicts slightly lower values of transmissivity. This can be explained by examining Fig. 5(a), which shows that for the case used in Fig. $6\left(L_{y}\right.$ at its maximum value), the transmissivity is slightly below its peak value. Second, it is interesting that the finite value of $L_{y}$ exercises some influence on the variation of $T_{x}$ with $L_{x}$, as implied by the pronounced oscillations observed in Fig. 6(a). Finally, the mode-matching solution does not predict the increase in extinction with aperture size, since this 2-D analysis does not allow for the depolarization ef- 
fects highlighted in Fig. 7. This fact also accounts for the large increase in extinction observed in Fig. 8(b) when the fill factor increases from $50 \%$ to $75 \%$.

\section{B. Polarizer Thickness}

All of the results shown above assume a polarizer thickness of $D=0.05 \lambda$. However, in practical polarizer configurations, the wire aspect ratio (and therefore the thickness) is an issue that impacts both the performance ${ }^{33}$ and the fabrication of the device. Indeed, intuition suggests that improved extinction can be obtained by increasing the conducting film thickness, since this should significantly reduce the transmissivity of the $y$ polarization while only slightly modifying the transmissivity for the $x$ polarization. Figure 9 shows the transmissivity and the extinction ratio for a square aperture with a fill factor of $50 \%$ for three different film thicknesses. Contrary to our hypothesis, however, the extinction ratio does not significantly increase with polarizer thickness. This is again due to the fact that the transmissivity $T_{y}$ is dominated by scattering into the $x$ polarization and therefore does not drop off significantly with increasing $D$. Figure 10(a) shows the extinction ratio for a $25 \%$ fill factor, illustrating that for cases where the value of $T_{y}$ is predominantly determined by the transmission of the $y$-polarized field, our hypothesis is confirmed. The results in Fig. 10(b), which show the predictions from the 2-D mode-matching technique, further support this conclusion.

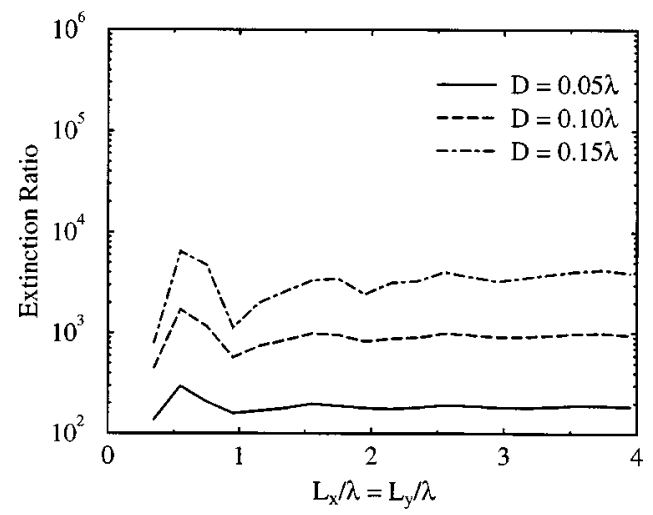

(a)

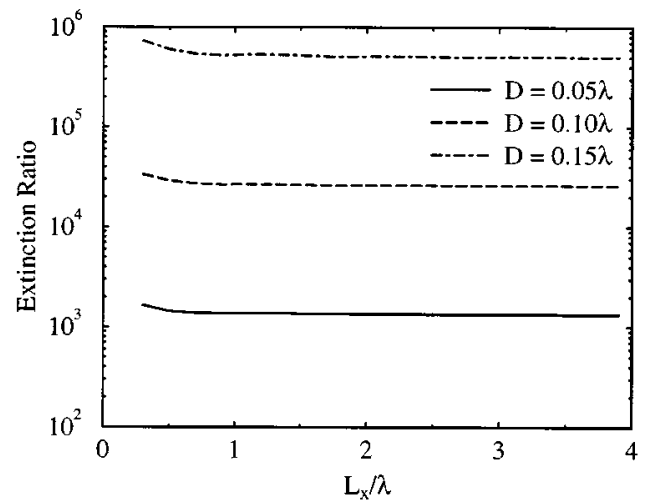

(b)

Fig. 10. Extinction ratio as a function of aperture side length for several values of thickness: (a) square aperture with $25 \%$ fill factor and (b) 2-D aperture $\left(L_{y} \rightarrow \infty\right)$ with $50 \%$ fill factor.
Table 1. Refractive-Index and Relative Permittivity Values for Different Metals at $\lambda=4 \mu \mathrm{m}$

\begin{tabular}{lcr}
\hline \multicolumn{1}{c}{ Metal } & Refractive Index & Relative Permittivity \\
\hline Molybdenum $^{a}$ & $5.16-j 10.16$ & $-76.59-j 104.87$ \\
Aluminum $^{b}$ & $6.76-j 40.96$ & $-1632.04-j 553.70$ \\
Gold $^{b}$ & $1.41-j 30.51$ & $-928.87-j 86.04$ \\
\hline
\end{tabular}

${ }^{a}$ Values taken from ellipsometry measurements.

${ }^{b}$ Values taken from the literature. ${ }^{31}$

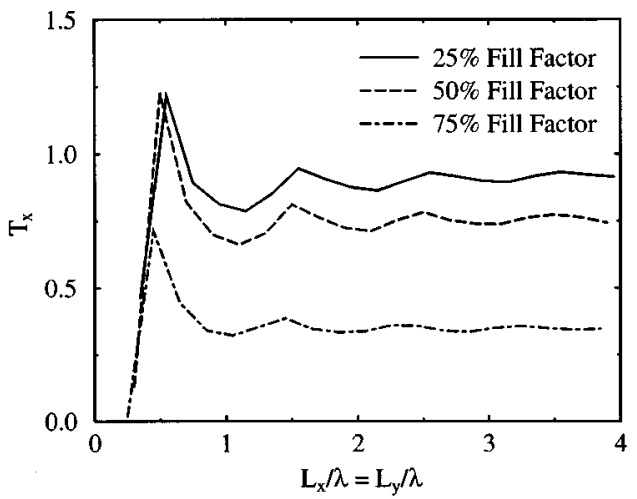

(a)

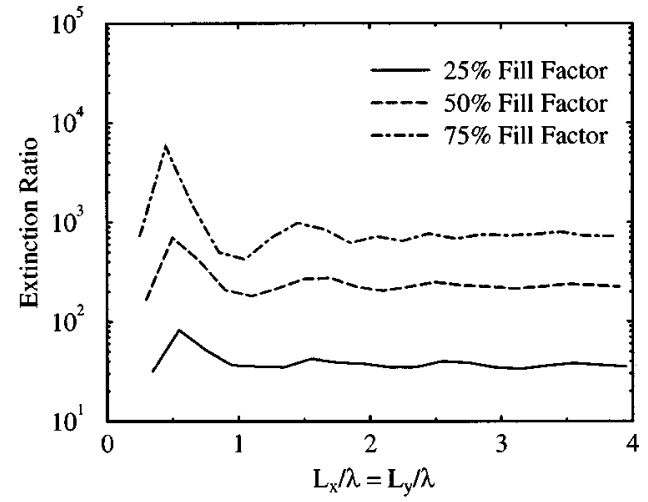

(b)

Fig. 11. (a) Transmissivity and (b) extinction ratio as a function of aperture side length for a square aperture for several values of fill factor $(D=0.05 \lambda)$ when the metal parameters are those of molybdenum.

\section{Polarizer Material}

Because practical polärizers are fabricated by using materials with finite conductivity, it is interesting to explore the impact of using realistic material models on the polarizer performance. In this case, however, only the wires are modeled by using the material parameters while the supporting ground plane remains perfectly conducting. This eliminates the transmission that would pass through a nonperfectly conducting ground plane and therefore produce ambiguous results when trying to assess the polarizer behavior. Table 1 shows the material parameters used in this study.

To assess the performance differences resulting from using these materials, we duplicate the results of Figs. 3 and 9 in Figs. 11 and 12, respectively, when the wires are replaced with molybdenum. At once, we notice very similar behaviors in the optical throughout between the 
perfectly and nonperfectly conducting cases. However, the finite-conductivity model predicts lower extinction ratio values, consistent with the notion that some transmission passes directly through the wire material. Additionally, we note that there is a much larger increase in extinction when moving from $50 \%$ to $75 \%$ fill factor or from $D=0.10 \lambda$ to $0.15 \lambda$ than what was observed for the perfectly conducting wires. This is due to the fact that transmission of the $y$-polarized field through the wires plays a significant role in the value of $T_{y}$, and this value is reduced as the fill factor or the thickness is increased. Figure 13 shows the effect of using different material parameters on the transmissivity and the extinction ratio. As can be seen, use of these materials exercises a noticeable impact on the polarizer performance.

\section{Practical Implications}

Many applications require both large extinction ratio and high optical throughput. Based on the above results, the extinction ratio can, in general, be augmented by increasing the fill factor, the thickness, or both, until reaching the limit imposed by cross-polarization scattering. Cross-polarization scattering can in turn be reduced by increasing the polarizer aperture (within the constraints of application requirements). A high optical throughput is achieved with a low fill factor or, if the fill factor must be large, by decreasing the polarizer thickness. Both of these cases affect the optical throughput. Clearly, designing a wire grid polarizer for a particular application

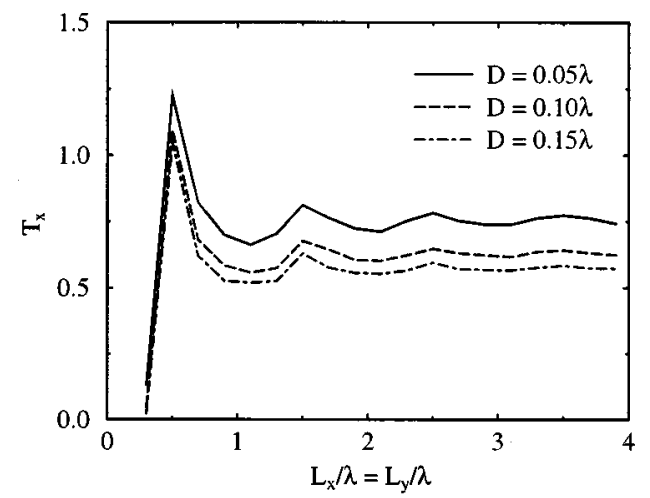

(a)

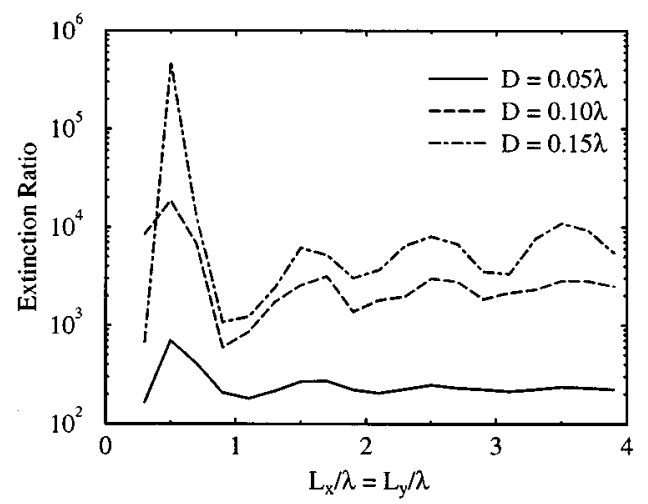

(b)

Fig. 12. (a) Transmissivity and (b) extinction ratio as a function of aperture side length for a square aperture for several values of thickness (50\% fill factor) when the metal parameters are those of molybdenum.

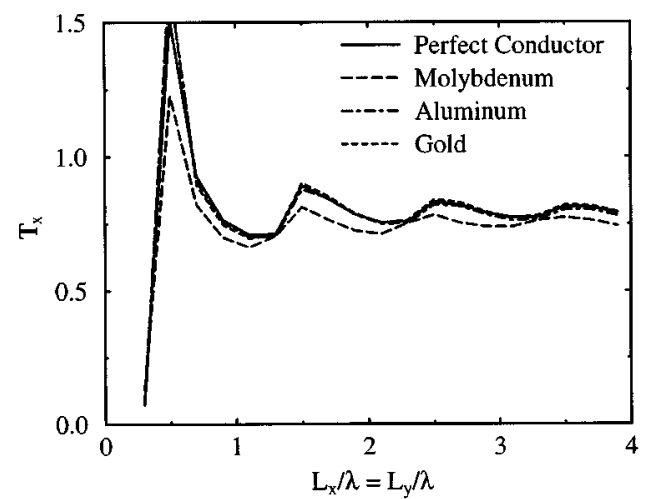

(a)

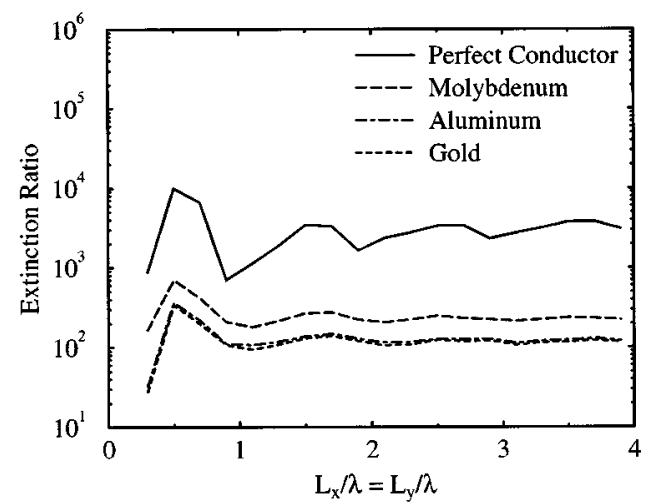

(b)

Fig. 13. (a) Transmissivity and (b) extinction ratio as a function of aperture side length for a square aperture for several different types of metal ( $50 \%$ fill factor, $D=0.05 \lambda$ ).

involves distinct trade-offs between the extinction ratio and the aperture size, and the extinction ratio and the optical throughput.

Fabrication considerations impose additional constraints on wire grid polarizer design. For example, consider the use of microfabrication techniques to create a polarizer for the infrared or the visible portion of the spectrum. Minimum feature size limitations determine the maximum slit density and also affect the achievable fill factor, both of which in turn influence the extinction ratio. Whether the wires are formed with an etch ${ }^{8,32}$ or a lift-off process, wire aspect ratio considerations affect the selection of polarizer thickness. Hence wire grid polarizer design involves making careful trade-offs between application requirements and physical and fabrication constraints.

\section{SUMMARY}

This paper has provided a detailed examination of the transmission characteristics of finite-aperture wire grid polarizers. The basic analysis approach uses the FDTD electromagnetic simulation method, with supporting data derived from a 2-D spectral-domain mode-matching technique. The FDTD implementation allowed modeling of real metals whose permittivity is characterized by a negative real part. These analysis tools were used to investigate the device transmissivity and extinction ratio for a wide variety of geometrical and material configurations. 
The studies revealed that the aperture can exercise a significant influence on the device transmission behavior. Most notably, it was found that in many structures, the extinction of the undesired polarization is limited by depolarizing scattering. Additionally, we observed that in general the transmissivity decreases and the extinction ratio increases with increasing fill factor or thickness. This in turn leads to a trade-off between the achievable optical transmission and extinction ratio. Finally, it was shown that using realistic metal parameters for the polarizer wires reduces the extinction ratio but does not significantly impact the optical throughput. Directions for future research include examining the effects of fabricationrelated defects and nonuniformities such as sidewall roughness and fill factor variations.

\section{ACKNOWLEDGMENTS}

G. P. Nordin acknowledges support by National Science Foundation CAREER Award ECS-9625040 and grant EPS-9720653.

Address correspondence to Michael A. Jensen at the location on the title page or by e-mail, jensen@ee.byu.edu.

\section{REFERENCES}

1. G. R. Bird and M. Parrish, Jr., "The wire grid as a nearinfrared polarizer," J. Opt. Soc. Am. 50, 886-891 (1960).

2. J. B. Young, H. A. Graham, and E. W. Peterson, "Wire grid infrared polarizer," Appl. Opt. 4, 1023-1026 (1965).

3. M. G. Moharam and T. K. Gaylord, "Rigorous coupled-wave analysis of metallic surface-relief gratings," J. Opt. Soc. Am. A 3, 1780-1787 (1986).

4. E. N. Glytsis and M. G. Moharam, "Rigorous coupled-wave analysis and applications of grating diffraction," in Diffractive and Miniaturized Optics, S. H. Lee, ed. (SPIE Press, Bellingham, Wash., 1993), Vol. CR49, p. 3-31.

5. M. G. Moharam, E. B. Grann, D. A. Pommet, and T. K. Gaylord, "Formulation for stable and efficient implementation of the rigorous coupled-wave analysis of binary gratings," J. Opt. Soc. Am. A 12, 1068-1076 (1995).

6. E. Chen and S. Y. Chou, "A novel device for detecting the polarization direction of linear polarized light using integrated subwavelength gratings and photodetectors," IEEE Photonics Technol. Lett. 9, 1259-1261 (1997).

7. J. Guo and D. J. Brady, "Fabrication of high-resolution micropolarizer arrays," Opt. Eng. 36, 2268-2271 (1997).

8. G. P. Nordin, J. T. Meier, P. Deguzman, B. Barbour, and M. W. Jones, "Arrays of infrared micropolarizers," in Diffractive Optics and Micro-Optics, Vol. 10 of 1998 OSA Technical Digest Series (Optical Society of America, Washington, D.C., 1998), pp. 133-135.

9. G. P. Nordin, J. T. Meier, P. C. Deguzman, and M. W. Jones, "Micropolarizer array for infrared imaging polarimetry," J. Opt. Soc. Am. A 16, 1184-1193 (1999).

10. K. Hirayama, E. N. Glytsis, and T. K. Gaylord, "Rigorous electromagnetic analysis of diffraction by finite-number-ofperiods gratings," J. Opt. Soc. Am. A 14, 907-917 (1997)

11. K. Hirayama, E. N. Glytsis, T. K. Gaylord, and D. W. Wilson, "Rigorous electromagnetic analysis of diffractive cylindrical lenses," J. Opt. Soc. Am. A 13, 2219-2231 (1996).

12. D. W. Prather, M. S. Mirotznik, and J. N. Mait, "Boundary integral methods applied to the analysis of diffractive optical elements," J. Opt. Soc. Am. A 14, 34-43 (1997).

13. E. N. Glytsis, M. E. Harrigtan, K. Hirayama, and T. K.
Gaylord, "Collimating cylindrical diffractive lenses: rigorous electromagnetic analysis and scalar approximation," Appl. Opt. 37, 34-43 (1998)

14. D. W. Prather, J. N. Mait, M. S. Mirotznik, and J. P. Collins, "Vector-based synthesis of finite aperiodic subwavelength diffractive optical elements," J. Opt. Soc. Am. A 15, 1599-1607 (1998)

15. A. Wang and A. Prata, Jr., "Lenslet analysis by rigorous vector diffraction theory," J. Opt. Soc. Am. A 12, 1161-1169 (1995).

16. M. Schmitz and O. Bryngdahl, "Rigorous concept for the design of diffractive microlenses with high numerical apertures," J. Opt. Soc. Am. A 14, 901-906 (1997).

17. Y.-K. Kok, "General solution to the multiple-metallicgrooves scattering problem: the fast-polarization case," Appl. Opt. 32, 2573-2581 (1993).

18. Y. S. Kim, H. J. Eom, J. W. Lee, and K. Yoshitomi, "Scattering from multiple slits in a thick conducting plane," Radio Sci. 30, 1341-1347 (1995).

19. S. H. Kang, H. J. Eom, and T. J. Park, "TM-scattering from a slit in a thick conducting screen: revisited," IEEE Trans. Microwave Theory Tech. 41, 895-899 (1993).

20. K. S. Yee, "Numerical solution of initial boundary value problems involving Maxwell's equations in isotropic media," IEEE Trans. Antennas Propag. AP-14, 302-307 (1966).

21. A. Taflove, Computational Electrodynamics: The FiniteDifference Time-Domain Method (Artech House, Boston, 1995).

22. M. A. Jensen and Y. Rahmat-Samii, "Performance analysis of antennas for hand-held transceivers using FDTD," IEEE Trans. Antennas Propag. 42, 1106-1113 (1994).

23. M. A. Jensen and G. P. Nordin, "Finite-aperture wire grid polarizers," Tech. Rep. 99-08 (Brigham Young University Microwave Earth Remote Sensing Laboratory, Provo, Utah, 1999) (http://www.ee.byu.edu/ee/mers/MERS_reports .html).

24. O. M. Mendez, M. Cahilhac, and R. Petit, "Diffraction of a two-dimensional electromagnetic beam wave by a thick slit pierced in a perfectly conducting screen," J. Opt. Soc. Am. 73, 328-331 (1983).

25. J. B. Judkins and R. W. Ziolkowski, "Finite-difference timedomain modeling of nonperfectly conducting metallic thinfilm gratings," J. Opt. Soc. Am. A 12, 1974-1983 (1995).

26. Z. P. Zhang, D. Y. Chu, S. L. Wu, W. G. Bi, R. C. Tiberio, R. M. Joseph, A. Taflove, C. W. Tu, and S. T. Ho, "Nanofabrication of 1-D photonic bandgap structures along a photonic wire," IEEE Photonics Technol. Lett. 8, 491-493 (1996).

27. K. M. Dridi and A. Bjarklev, "Optical electromagnetic vector-field modeling for the accurate analysis of finite diffractive structures of high complexity," Appl. Opt. 38, 1668-1676 (1999)

28. H. Y. D. Yang, "Finite difference analysis of 2-D photonic crystal," IEEE Trans. Microwave Theory Tech. 44, 26882695 (1996).

29. J.-P. Berenger, "A perfectly matched layer for the absorption of electromagnetic waves," J. Comput. Phys. 114, 185200 (1994).

30. D. S. Katz, E. T. Thiele, and A. Taflove, "Validation and extension to three dimensions of the Berenger PML absorbing boundary condition for FD-TD meshes," IEEE Microwave Guid. Wave Lett. 4, 268-270 (1994).

31. D. R. Lide, ed., CRC Handbook of Chemistry \& Physics, 73rd ed. (CRC Press, Boca Raton, Fla., 1992).

32. B. Stenkamp, M. Abraham, W. Ehrfeld, E. Knapek, M. Hintermaier, M. T. Gale, and R. Morf, "Grid polarizer for the visible spectral region," in Nanofabrication Technologies and Device Integration, W. Karthe, Proc. SPIE 2213 288-296 (1994).

33. H. Lochbihler and R. Depine, "Diffraction from highly conducting wire gratings of arbitrary cross-section," J. Mod. Opt. 40, 1273-1298 (1993). 